\title{
What is top-down about seeing enemies? Social anxiety and attention to threat
}

\author{
Hannah L. Delchau ${ }^{1} \cdot$ Bruce K. Christensen ${ }^{1} \cdot{\text { Richard } \text { O'Kearney }^{1} \cdot \text { Stephanie C. Goodhew }}^{1}$
}

Published online: 2 December 2019

(C) The Psychonomic Society, Inc. 2019

\begin{abstract}
An attentional bias to threat is an important maintaining and possibly aetiological factor for social anxiety. Despite this, little is known about the underlying mechanisms of threat biases, such as the relative contributions of top-down and bottom-up attention. In order to measure attentional bias toward threat, the current study employed a variation of the dot-probe task in which participants' attention was initially cued to the left or right side of the screen before an angry face paired with a neutral face was displayed, and subsequently participants responded to a probe in the locus of one of the faces. This design provides separate measures of engagement with and disengagement from threat. In addition, in order to manipulate the availability of top-down attentional resources, participants completed this task under no, low (simple arithmetic task), and high (difficult arithmetic task) working memory load. Higher levels of social anxiety were found to be associated with increased engagement with threat under no-load, whereas this effect was eliminated under low-load and high-load conditions. Moreover, social anxiety was not associated with delayed disengagement from threat. These results highlight the critical role of top-down attention for engaging attention with threat.
\end{abstract}

Keywords Selective attention · Spatial attention · Working memory load · Social anxiety · Dot-probe · Threat bias; Top-down; Bottom-up

Faces convey critical social information. It is, therefore, unsurprising that social anxiety, which is characterized by a fear of being judged and scrutinized by others, is associated with an attentional bias toward threatening facial expressions (e.g., anger and disgust). For example, Rapee and Heimberg (1997) hypothesized that socially anxious individuals vigilantly monitor their external environment for signs of negative evaluation from others. This results in a reinforcing loop of anxiety, whereby socially anxious individuals are more likely to notice signs of disapproval from others, which further increases their anxiety, causing them to be more hypervigilant of criticism. This model is supported by research findings that socially anxious individuals show biased attention toward photos of faces depicting angry, hostile, and disgust expressions compared with neutral facial expressions (Mogg, Philippot, \& Bradley, 2004; Pishyar, Harris, \& Menzies, 2004), and that correcting this bias can reduce symptoms of anxiety ( $\mathrm{Liu}, \mathrm{Li}$,

Stephanie C. Goodhew

stephanie.goodhew@anu.edu.au

1 Research School of Psychology, The Australian National University, Building 39, Canberra 2601, Australia
Han, \& Liu, 2017). Given the strong link between attention and social anxiety, it is imperative that research is conducted to improve our understanding of the mechanisms underlying biased attention to threat. The purpose of this study, therefore, was to apply a key theoretical framework of attention to understand the nature of biased attention to threat for socially anxious individuals.

A central tenet of the current study is that taxonomies of normative attention may guide our understanding of anxietyrelated attention to threat. In this vein, it is commonly accepted that at any given moment, there is typically far more information available in visual scenes than the human brain is capable of fully processing. The attentional system, which acts like a filter, is crucial for allowing us to process and interact with our visual world. That is, the attentional system selects important and relevant information for further processing at the expense of other information (Desimone \& Duncan, 1995; Kastner \& Pinsk, 2004). The manner in which selective attention takes place can be subdivided into top-down and bottom-up attention. Top-down attention refers to the voluntary allocation of attention toward particular objects, features, or spatial locations based on one's current goals. For example, when searching for your child on a busy beach, if you know that 
he or she is wearing a blue swimsuit, you can more efficiently search to find your child by selectively attending to blue objects. Bottom-up (also known as automatic) attention, by contrast, is an involuntary, rapid, and inflexible process that selects visual information based on the salience of the stimulus features (e.g., automatically orienting to a seagull that swoops down in front of you).

A general question emerging from the anxiety-attention literature has been whether threat detection relies on bottomup or top-down attention. The argument that attention to threat is driven by bottom-up mechanisms comes from an evolutionary perspective, which emphasizes the adaptive purpose of attending to threat (Kenrick, Neuberg, Griskevicius, Becker, \& Schaller, 2010; Lang, Bradley, \& Cuthbert, 1997; LeDoux, 1996; LoBue, Rakison, \& DeLoache, 2010; Mogg \& Bradley, 1998; Öhman, 2007). This argument contends that survival can depend on the rapid detection of threatening stimuli that might cause an individual harm (e.g., predators, dangerous environments, social signs of anger from other people). The bottom-up attentional system is ideally suited to the purpose of triaging the processing of such stimuli, since it is more rapid than the top-down attentional system (Busse, Katzner, \& Treue, 2008), and a timely response to threat is of the essence. Furthermore, its operation is automatic and therefore less dependent on the individual's intentions or competing goals at that point in time. In other words, it is a more fail-safe system.

The bottom-up system typically operates at the level of basic stimulus features (e.g., colour, shape, orientation, motion). From this point of view, it would be difficult for the system to be tuned to a more complex property, such as "threat," which can take varied visual forms and is undoubtedly multifactorial in nature. However, consistent with the notion of a bottom-up attentional system tuned to features of threat, a body of research suggests that such a bias is driven by specific low-level perceptual features associated with prototypical threatening stimuli (LoBue, 2014; LoBue \& DeLoache, 2011; LoBue \& Larson, 2010). One example of this is a bias toward curvilinear shapes, which are reminiscent of the shape of a snake or spider's legs. In a visual search task, LoBue (2014) demonstrated that participants have a bias toward curvilinear shapes compared with rectangular shapes. Furthermore, illustrating the association between anxiety and threat detection, LoBue also showed that fear-inducing film clips facilitate the detection of curvilinear shapes. Furthermore, children display biases toward downward "V" and triangle shapes, which are similar to the shape of angry eyebrows (LoBue \& Larson, 2010). This adds further support to the notion that threat detection may be an evolved, automatic system.

However, an accumulating body of research indicates that threat detection may be modulated by, or even driven by, topdown attention and is not purely the product of bottom-up attention. Conceptually, this means that rather than an automatic (possibly even innate) bias toward threat that is triggered as reflexively as orienting toward a bright flash of light, the bias toward threat might reflect top-down attention. Top-down attention can work at a more conceptual level, such as the level of semantic categories (Goodhew, Kendall, Ferber, \& Pratt, 2014; Most, 2013; Wyble, Folk, \& Potter, 2013), which could arguably include the label "threat." The operation of top-down attention is more volitional, flexible, and contextspecific than bottom-up biases. Thus, if the bias toward threat in socially anxious individuals occurs via a top-down attentional route, it should be susceptible to a number of contextual factors, such as the individual's goals. In contrast, if the bias toward threat is a bottom-up process, it should be more invariant to these influences.

In an attempt to disentangle whether the attentional bias predominately operates via a top-down or a bottom-up attentional system, previous research has used working memory (WM) load tasks. WM is a limited-capacity system, which, in the face of continued processing and distraction, is responsible for the active maintenance of information (Conway et al., 2005). More specifically, WM functions include the storage and rehearsal of information and executive functions, with tasks tapping these functions including counting, operation span, and reading span (Conway et al., 2005). Indeed, recent researchers have argued that WM is indistinguishable from cognitive control (i.e., top-down attention; Qi et al., 2014), as the two functions overlap in neural activity (e.g., Bunge, Ochsner, Desmond, Glover, \& Gabrieli, 2001; Kondo, Osaka, $\&$ Osaka, 2004). Tasks requiring bottom-up attention are unaffected by WM load (Jonides, 1981), whereas WM, which is essential for keeping goals in mind, affects tasks that require top-down attention (de Fockert, Rees, Frith, \& Lavie, 2001; Downing, 2000). A logical way to test if an attentional task requires top-down resources is to measure if attention toward threat is altered by the addition of a WM load. Theoretically, when the demands of the WM task increase, the available resources for the second task decrease (Sarampalis, Kalluri, Edwards, \& Hafter, 2009; Tun, McCoy, \& Wingfield, 2009). The extent to which performance on the secondary task decreases provides clues as to the role of WM demanded by the primary task (Kerr, 1973). Thus, if an attentional bias toward threat is unaffected by a WM load, it suggests that the task requires few top-down attentional resources and is predominantly driven by bottom-up attention. However, if an attentional bias is attenuated under high load, it indicates that the bias is driven significantly by top-down attention (note that the predictions of WM load and perceptual load differ; see Lavie, 2005).

Van Dillen and Koole (2009) tested this by using a gendernaming task with centrally presented happy and angry faces. They found that angry faces resulted in slower gender naming, compared with happy faces, under low-load but not high-load conditions. This indicates that participants processed the 
emotion of the faces only when spare attentional resources were available, and the threat bias required top-down attention. Although this is an important finding, this research only tested attention to centrally presented faces and did not test the association with anxiety. However, shifts of attention across space are crucial to the conceptualization of the threat biases for socially anxious individuals, because in everyday life there is competition between visual stimuli and threat-related stimuli, and these stimuli do not always appear in the current locus of attention. For example, to explain why someone with social anxiety attends to threatening facial expressions but not to encouraging expressions from the audience when presenting a speech requires understanding shifts in spatial attention toward and away from facial expressions.

The relationship between social anxiety and spatial shifts of attention toward threat was assessed by Judah, Grant, Lechner, and Mills (2013). Two groups of participants were included: a socially anxious group and a nonanxious control group. These researchers employed a dot-probe task and presented participants with images of happy, disgust, and neutral facial expressions under no-WM-load, low-WM-load, and high-WM-load conditions. In this design, two faces were presented on each trial; one to the left and one to the right of fixation. A probe (e.g., a letter) followed in the location of one of the faces, and participants reported its identity. Faster reaction times (RTs) to the probe following a threatening facial expression compared with RTs to a probe following a neutral facial expression indicated that participants were attending to the threatening face. This difference in RT reflected a threat bias.

Judah et al. (2013) found that the group with social anxiety had a reduced bias to the disgust expressions under the noload, and a greater bias toward the disgust expressions under high-load conditions, compared with the control group. Since these researchers employed long presentation times for the faces $(1,000 \mathrm{~ms})$, they argued that this reflected avoidance of threat under no-load conditions and delayed disengagement from threat under high-load conditions.

In other words, when the social anxiety group had attentional resources to spare (no-load condition), they could volitionally shift their attention to avoid the threatening face. However, when these resources were consumed with the WM-load task, they were not able to instantiate this disengagement. One potential issue with this design is that it assumes that all participants initially shifted their attention toward the threatening stimulus to an equal degree. If, however, social anxiety affected participants' initial engagement with threat, then subsequent measures of disengagement and avoidance are undermined. It is, therefore, essential to measure engagement and disengagement biases separately.

Employing a paradigm developed by Grafton and MacLeod (2014) and Rudaizky, Basanovic, and MacLeod (2014), this issue was mitigated by Boal, Christensen, and
Goodhew (2018). Separately measuring attentional engagement and disengagement biases, these researchers tested whether the threat bias associated with social anxiety is driven by top-down or bottom-up attention. On each trial, participants were initially presented with a cue (a small red line oriented horizontally or vertically). This cue was presented either on the left or right side of the screen and, accordingly, secured participants' attention in the same location or opposite location to the target image. Participants then viewed a target image (an upright neutral or disgust face) paired with its inverted counterpart. A probe (a small red line oriented horizontally or vertically) was then presented in the locus of one of the images, and participants reported whether it matched the orientation of the initial cue or was different. A disengagement trial was defined as a trial in which the target face was presented in the same location as the preceding cue. On half of these trials, the probe was then presented in the same location as the target face, and on half of the trials the probe was presented in the opposite location. The disengagement condition measured the speed to which participants disengaged their attention from the target face to respond to a subsequent probe presented in the opposite location, compared with when they were required to respond to a probe in the same location as the target face. Therefore, slower disengagement of attention on the threat-target trials compared with the neutral-target trials indicates a disengagement bias for threat. An engagement trial, by contrast, was defined as a trial in which the target face was presented in the opposite location to the preceding cue. On half of these trials, the probe was then presented in the same location as the target face, and on half of the trials the probe was presented in the opposite location. The engagement trials measured the speed to which participants shifted their attention away from the inverted face toward the target face. Faster RTs to the probe following the target face compared with the probe following the inverted face for threateningtarget trials compared with neutral-target trials indicated a threat bias.

To test if attentional biases to threat requires top-down attentional resources, the task was performed under three WMload conditions: the no-load, low-load (one-digit memory task), and high-load (six-digit memory task) conditions. Surprisingly, these researchers found that high social anxiety was associated with an engagement bias away from threat, whereas low social anxiety was associated with a bias toward threat. The disengagement bias was not related to social anxiety. Finally, since these effects were not significantly affected by the WM-load manipulation, it was concluded that engagement with threatening facial expressions had minimal contributions from top-down mechanisms.

The present study aimed to extend on the research of Boal et al. (2018) by overcoming three limitations of their study. Firstly, the study employed inverted faces as control images (rather than abstract art images, which were used by Grafton 
\& Macleod, 2014, and Rudaizky et al., 2014). This approach assumes that inversion disrupts the emotion processing of faces, which was predicated on the basis that inversion substantially impairs configural processing of faces (de Gelder, Teunisse, \& Benson, 1997; Searcy \& Bartlett, 1996). However, other research challenges this view, finding that rapid emotion detection can occur for inverted faces (Arnold \& Lipp, 2011). Although Boal and colleague's finding of reduced engagement with threatening faces may have reflected attentional avoidance (see Cisler \& Koster, 2010), it may also be due to differences in attending to the inverted face. For instance, it is possible that socially anxious participants, due to hypervigilance to threat, may have detected the threatening expressions contained in the inverted disgust faces and therefore readily shifted toward them. This could account for what appeared to be an attentional bias away from (upright) threat observed by Boal et al. (2018). To overcome this issue, the present study directly paired negative and neutral expressions on each trial rather than employing inverted faces (see Grafton \& MacLeod, 2016, for a similar approach to selecting distractor images).

Secondly, the present study changed the presentation time of the images from $500 \mathrm{~ms}$ to $200 \mathrm{~ms}$. Although $500 \mathrm{~ms}$ is a commonly used presentation time in the dot-probe literature, it allows for participants to make multiple covert and/or overt shifts of attention while the image is being presented. Therefore, initial shifts of attention are confounded with later attentional stages (e.g., avoidance). The present study displayed the faces for $200 \mathrm{~ms}$ to minimize the occurrence of multiple shifts of attention.

The third issue addressed was the complexity of the WM task. The present study employed an arithmetic task rather than memorizing digit strings, as was employed in Boal et al. (2018). It is possible that this manipulation had a limited impact on performance because it did not comprehensively exhaust WM resources. The Wechsler Adult Intelligence Scale-Fourth Edition (WAIS-IV) manual, describes digit span forward as a test involving "attention, encoding and auditory processing" (Wechsler, 2008, p. 15). Thus, the digit span task in Boal and colleagues' study may be better conceptualized as measuring attention and short-term memory rather than WM. Arithmetic, by contrast, has been found to involve all components of WM (DeStefano \& LeFevre, 2004).

\section{Present Study}

In sum, the aim of the present study was to determine whether engagement and disengagement of attention to threat for individuals with social anxiety is largely driven by top-down or bottom-up attention. To measure attentional biases, the present study employed a dot-probe task in which participants viewed a pair of faces (one angry and one neutral) on each trial. RT to a probe, which was presented either in the location of the preceding angry or neutral face on each trial, was measured. Cueing scores were calculated for the engagement and disengagement trials separately and were compared under three WM-load conditions: no-load, low-load, and high-load conditions. The addition of the WM load was designed to test whether attention toward threat requires top-down or bottom-up attention.

Based on Grafton and MacLeod's (2016) findings, under no-load and low-load conditions, it was expected that higher social anxiety would be associated with an attentional engagement bias toward the angry faces. If the engagement bias is largely driven by bottom-up attention, this bias would be unaffected by the load manipulation. ${ }^{1}$ However, if top-down attentional resources are required for participants to shift toward the angry faces, the engagement bias will reduce in the high-load condition. Finally, the study also planned to clarify whether social anxiety is associated with delayed attentional disengagement for short stimulus presentation times and, if it is, whether it is affected by WM load.

\section{Method}

\section{Participants and design}

One hundred and three participants ( 75 female and 28 male) were recruited from the Australian National University via online advertisement and the university electronic sign-up system. The sample size was chosen so as to be able to detect an effect size $f$ of $0.23\left(\eta_{\mathrm{p}}{ }^{2}=.05\right)$. This effect size was selected since Grafton and MacLeod (2016) reported an effect size of $\eta_{\mathrm{p}}{ }^{2}=.05$ for the interaction between social anxiety and the engagement bias. Using $\mathrm{G}^{*}$ Power calculations ${ }^{2}$ (ANOVA: repeated measures, between factors) with power of 0.8 and an effect size $f$ of 0.23 , the recommended sample size was 90 . Therefore, the sample size of 103 was deemed sufficient (so as to account for some exclusions in data, which were predicted to occur; note that after exclusions, 91 participants were

\footnotetext{
${ }^{1}$ An alternative prediction derives from load theory (Lavie, 2005). Load theory suggests that distractibility increases with increasing cognitive load. According to this theory, the attentional bias toward threat in individuals high in social anxiety should actually increase as load increases. Note, however, that we did not observe this pattern of results.

${ }^{2}$ Although the G*Power analysis did not include the covariate of social anxiety, this analysis with one two-level, between-subjects factor and a six-level, repeated-measures factor was deemed the closest approximation to this study's design. Since designing the study, it has come to our attention that G*Power does not, in fact, allow for the computation of power for designs with more than one repeated-measures factor, as was present here. When we specify six measurements, $G *$ Power assumes that these all reflect levels of one variable $(6$ $\times 1$ ), rather than a $2 \times 3$ factorial design. For future research, we plan to use the superior newly available calculators that permit these sorts of common experimental designs, in addition to permitting for correction for publication bias (Anderson, Kelley, \& Maxwell, 2017). However, since G*Power was used, this is what is reported here.
} 
included in the final analyses). Restrictions were that participants were Caucasian, ages 18-30 years, with normal vision or corrected-to-normal vision. Participants' ages ranged from 18 to 30 years $(M=19.74, S D=2.32)$, and eight participants were left-handed. Participants were offered course credit (if eligible) or \$10 cash (AUD).

\section{Experimental stimuli and apparatus}

This experiment was conducted in a dimly lit room. Viewing distance was set with a chin rest at $44 \mathrm{~cm}$, and a liquid crystal display monitor running at a $60 \mathrm{~Hz}$ refresh rate was used to display the stimuli. Stimuli were programmed in MATLAB using the Psychophysics Toolbox (Brainard, 1997). The background was set to black, and the arithmetic questions were presented in type size 18 white Helvetica font. Images of faces were taken from the NimStim database (Tottenham et al., 2009). The use of this database in this area of research is common (e.g., Alon, Arad, Pine, \& Bar-Haim, 2019; Dima, Perry, Messaritaki, Zhang, \& Singh, 2018; Wieckowski, Capriola-Hall, Elias, Ollendick, \& White, 2019; White, Capriola-Hall, Wieckowski, \& Ollendick, 2019). To limit variability in responding to faces due to race, the images selected were Caucasian faces, and participants were Caucasian (based on self-report). Caucasian images were employed to control for the cross-race effect in which other-race faces may be processed differently to faces of one's own race (for a review, see Young, Hugenberg, Bernstein, \& Sacco, 2012). Furthermore, closed-mouth neutral and angry expressions were selected to reduce bottom-up capture effects from teeth visibility (Horstmann, Lipp, \& Becker, 2012). Low-level differences were minimized by ensuring that the two face images presented on each trial were as similar as possible in all respect except their emotional expression. Several faces that met the above criterion were not included, because of issues intrinsic to the image set. One image was the incorrect size (Model 1), one had confounding facial hair (Model 31), and one did not have the full range of images available (Model 27). Therefore 22 models (seven females and 15 males) were included, each with three associated images of neutral and angry expressions. Although Boal et al. (2018) employed disgust expressions, the current study employed angry expressions because a greater number of closed-mouth angry faces were available in the NimStim database compared with closed-mouth disgust faces. Anger is a form of social threat, and therefore theoretically individuals with high levels of social anxiety should be preferentially sensitive to angry faces relative to neutral faces. Furthermore, previous research demonstrates empirically that socially anxious individuals display the same attentional bias toward angry and disgust expressions (Grafton \& MacLeod, 2016). During the experiment, each image subtended approximately $7.60^{\circ} \times 9.76^{\circ}$ of visual angle, with a gap of $7.11^{\circ}$ of visual angle between the two presented images.

\section{Questionnaires}

Symptoms of psychopathology were measured using two questionnaires. Firstly, to assess social anxiety, the Liebowitz Social Anxiety Scale (LSAS; Liebowitz, 1987) was employed in its self-report form. For each of the 24-items, participants are asked to rate both their fear and avoidance on a 4-point Likert-type scale. Fear is rated from 0 (none) to 3 (severe) and avoidance rated from 0 (never) to 3 (usually, $68 \%-100 \%$ ). A total score can be calculated by summing scores from both the fear and avoidance scales, yielding a maximum score of 144. This measure was selected as it has good psychometric properties (Baker, Heinrichs, Kim, \& Hofmann, 2002; Fresco et al., 2001; Levin, Marom, Gur, Wechter, \& Hermesh, 2002; Oakman, Van Ameringen, Mancini, \& Farvolden, 2003; Rytwinski et al., 2009) and is brief and easily administered. In a large university study, Caballo, Salazar, Irurtia, Arias, and Nobre (2013) reported that males had a mean score on the LSAS-Self Report (LSAS-SR) of 42.19 and females had a mean score of 45.73 . The current study had higher scores than those of Caballo et al. (2013), as total LSAS-SR scores ranged from 11 to $104(M=55.41, S D=$ 22.87), with the Fear subscale ranging from 5 to $56(M=$ 28.57, $S D=11.68)$ and Avoidance ranging from 4 to $54(M$ $=26.91, S D=12.16$ ).

Secondly, generalized anxiety and depression were assessed with the Depression Anxiety Stress Scale-21 (DASS-21), which is a short form of Lovibond and Lovibond's (2005) 42-item self-report measure (DASS). Although the DASS-21 results were not primary measures of interest, these data were collected to provide more complete demographic information of the participants. The DASS- 21 is a 21-item measure in which participants rate the degree to which symptoms applied to them over the past week. Ratings are made on a 4-point Likert scale from 0 (never) to 3 (almost always). This measure provides separate scores for depression, anxiety, and stress, each ranging from 0 to 42 . The DASS-21 was chosen because it has good convergent, discriminant, and construct validity (Crawford \& Henry, 2003; Henry \& Crawford, 2005; Lovibond \& Lovibond, 1995) and reliability (Antony, Bieling, Cox, Enns, \& Swinson, 1998; Brown, Chorpita, Korotitsch, \& Barlow, 1997; Crawford \& Henry, 2003; Lovibond \& Lovibond, 1995). The depression scores ranged from 0 to $38(M=10.17, S D=8.97)$, anxiety ranged from 0 to $38(M=9.59, S D=8.17)$, and stress ranged from 0 to $38(M=15.30, S D=8.82)$.

\section{Procedure}

Participants completed the consent form and demographic questions, the computer task, and then the questionnaires. The computer task consisted of three blocks of trials (no-load, low-load, and high-load trials), order counterbalanced across 
participants. Each block consisted of an initial 12 practice trials and then a further 112 experimental trials; 56 of those trials consisted of engagement trials, and 56 were disengagement trials (randomly intermixed).

On each trial, a blank screen was presented initially for $1,000 \mathrm{~ms}$. Next, two white rectangular outlines, one to the left and one to the right of fixation, were presented for $1,000 \mathrm{~ms}$. These rectangular outlines subtended $7.6^{\circ} \times 9.8^{\circ}$ of visual angle, and the width of the lines subtended approximately $0.09^{\circ}$ and remained visible while the cue was presented. A smaller red rectangle, subtending $1.9^{\circ} \times 2.4^{\circ}$, was also presented inside one of the white rectangles to indicate the location of the to-be-presented cue. The cue (a small red line) was then presented within the box for $200 \mathrm{~ms}$. This cue could be oriented horizontally or vertically and subtended a visual angle of $0.5^{\circ} \times 0.1^{\circ}$. The presentation of the cue on the left and right side of the screen were randomized with equal probability of it appearing in each location.

After these stimuli disappeared, two images of faces were presented for $200 \mathrm{~ms}$, one to the left and one to the right of fixation, such that they occupied the locations that the white rectangles previously occupied. On each trial, one face was angry and one was neutral, and the two expressions were from the same model. In addition, an equal proportion of female and male faces were presented in each condition. After these faces offset, a probe (a small red line) oriented horizontally or vertically, which was identical in appearance to the cue, was then presented in the locus of one of the faces and was oriented horizontally or vertically. The presentations of the cue and probe as horizontal or vertical were each randomized throughout the experiment. Moreover, for both the engagement and disengagement conditions, the probe appeared behind the angry face on $50 \%$ of the trials and behind the neutral face on $50 \%$ of the trials (randomly intermixed). Participants made a keyboard press to report whether the orientation of the probe matched the orientation of the cue as quickly and accurately as possible. RTs were measured as the time between onset of the probe and the participants' key press.

The low-load and high-load conditions also included a WM load that sandwiched the dot-probe task. On the lowload and high-load trials, after the initial presentation of the blank screen for 1,000 ms, an additional arithmetic task was presented. At the beginning of each trial, an arithmetic question was presented centrally for $2,500 \mathrm{~ms}$. In the low-load condition, the question followed the format "A + B" (simple question) and, in the high-load condition, the question followed the format " $\mathrm{A}+\mathrm{B}-\mathrm{-}+\mathrm{C}$ " (difficult question) (where A, $\mathrm{B}, \mathrm{C}$, and $\mathrm{D}$ consisted of randomly generated whole numbers ranging from 1 to 10 , with a restriction that the answer could only be a positive integer). At the end of each trial, after participants responded to the probe, the words "Even or Odd" was presented centrally. Participants then made a key press to indicate whether the answer to the arithmetic question was an even ("z") or odd number (“"/) (see Fig. 1).

The presentation time for the arithmetic question was selected based on pilot testing. The pilot testing sample consisted of 10 participants, ages 18-30 years, with normal vision or vision corrected with glasses. The mean age was 23.7 years $(S D=3.6)$; eight participants were female and two were male, and all were right-handed. During pilot testing, participants completed a 20 -trial block of "simple questions" and a 20-trial block of "difficult questions," and were asked to make keyboard presses to respond to whether the question was an even number or an odd number. For the simple question block, mean accuracy was $95.3 \%(S D=6.8 \%)$ and RTs were $1351.2 \mathrm{~ms}(S D$ $=311.9 \mathrm{~ms}$ ). By comparison, for the difficult question block, mean accuracy was $92.9 \%(S D=5.3 \%)$ and mean

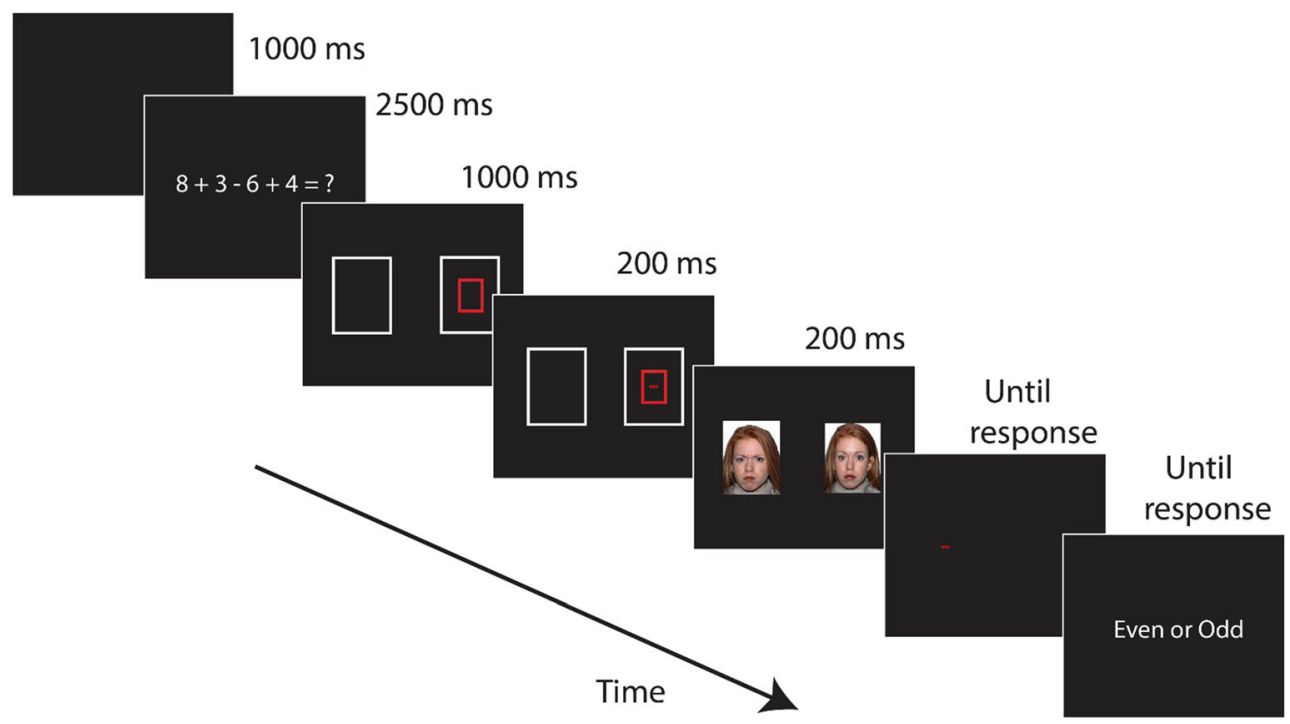

Fig. 1 A schematic of an engagement trial under high load 
RTs were $5526.2 \mathrm{~ms}(S D=1479.0 \mathrm{~ms})$. These findings confirmed that participants, on average, would not be able to solve the difficult arithmetic question prior to the faces being presented in the high-load arithmetic condition. Therefore, participants' WM resources would be consumed during face presentation.

Planned calculations In accordance with Grafton and Macleod's (2016) method, an engagement trial was defined as a trial in which the negative face was presented in the opposite location of the preceding cue. This is because these trials measure the likelihood that participants will shift their attention toward the negative face. By contrast, for disengagement trials, the negative face was presented in the same location as the preceding cue. Therefore, participants were required to disengage their attention from the negative face to respond to a subsequent probe in the opposite location. Higher scores for the attentional engagement bias index reflected facilitated attentional orienting toward the angry expression. More specifically, scores above zero represented faster responding on trials where participants had to shift their attention from the location of the neutral face to the location of the angry face than trials in which they were required to maintain their attention at the location of the neutral face. Furthermore, as reported previously, for the engagement conditions, the probe appeared behind the angry face on $50 \%$ of the trials and behind the neutral face on $50 \%$ of the trials. The equation is as follows:

\section{Attentional Engagement Bias Index}

$=($ Cue in locus of neutral facial image : $)$ RT for probe in locus of neutral facial image minus (Cue in locus of neutral facial image :) $\mathrm{RT}$ for probe in locus of negative facial image.

Similarly, higher scores for the attentional disengagement bias index reflected greater difficulty disengaging from the angry expression. More specifically, scores above zero represented faster responding on trials where participants maintained their attention to the location of the angry face compared with trials when they were required to shift their attention to the opposite location. In addition, the probe appeared behind the angry face on $50 \%$ of the trials and behind the neutral face on $50 \%$ of the trials. The equation is as follows:

\section{Attentional Disengagement Bias Index}

$=($ Cue in locus of negative facial image : $)$

RT for probe in locus of neutral facial image minus (Cue in locus of negative facial image:) $\mathrm{RT}$ for probe in locus of negative facial image.

\section{Results}

One participant's data were excluded because they did not meet the criteria for being Caucasian, eight participants' data were excluded because they were close to chance-level $(<60 \%)$ accuracy on the arithmetic task, and a further three participants' data were excluded because their RTs were slower than 3.29 standard deviations from average. Therefore, 91 participants' data were included in further statistical analyses. Raw data are available here (https://osf.io/ bex6q/).

Mean accuracy on the probe task was $95.7 \%(S D=4.0 \%)$ in the no-load condition, $97.1 \%(S D=3.7 \%)$ in the low-load condition, and $94.1 \%(S D=5.0 \%)$ in the high-load condition. $^{3}$ Mean accuracy on the arithmetic task was $88.6 \%$ (SD $=10.4 \%)$ in the low-load condition and $85.6 \%(S D=8.8 \%)$ on the high-load condition. While correct RT was the primary measure of interest in this study, to ensure that there were no speed-accuracy trade-offs, accuracy data were also analyzed. To this end, a 2 (trial type: engagement or disengagement) $\times 3$ (load: no, low, or high) or 2 (probe: locus of negative face or locus of neutral face) ANCOVA on probe identification accuracy with the continuous predictor variable of social anxiety was conducted. Social anxiety scores were centered on the grand mean to reduce multicollinearity and improve interpretability (see Tabachnick \& Fidell, 2013). Violations in sphericity were corrected with the Greenhouse-Geisser estimate. A significant effect was found for load, $F(1.72,153.43)=15.91$, $p<.001, \eta_{\mathrm{p}}{ }^{2}=.152$. No other significant main or interaction effects were found ( $p \geq .093$ ), indicating that accuracy did not differ based on participants' or the probe location. Follow-up analyses consisted of ANCOVAs concurrently comparing two load conditions (averaged across the other factors) with social anxiety as a covariate. These revealed that accuracy for the probe was significantly higher in the low-load condition compared with both the no-load condition, $F(1,89)=10.96, p=$ $.001, \eta_{\mathrm{p}}{ }^{2}=.110$, and the high-load condition, $F(1,89)=$ $27.00, p<.001, \eta_{\mathrm{p}}{ }^{2}=.233$. In addition, accuracy was significantly higher in the no-load condition compared with the high-load condition, $F(1,89)=7.70, p=.007, \eta_{\mathrm{p}}{ }^{2}=.080$. Therefore, participants had the highest accuracy on the lowload condition, followed by the no-load condition, and then the high-load condition.

Exclusions were made for trials in which RTs were less than $100 \mathrm{~ms}$ or greater than 2.5 standard deviations above the individual participant's mean RT for each load condition

\footnotetext{
${ }^{3}$ Note that the means reported here are derived from taking the average accuracy for the angry and neutral probe trials. These differ slightly from the aggregate mean scores provided in the raw data file, as those were computed prior to trial assignment (i.e., mean across all trials of this type, rather than mean of angry and neutral condition trials of this type). The means reported in text can be verified from the available raw data, by taking the average accuracy for the angry and neutral trials at each load.
} 
separately. The percentage of excluded trials based on RT outliers were $2.7 \%, 3.1 \%$, and $3.1 \%$ for the no-load, low-load, and high-load load conditions, respectively. Data from trials in which participants performed incorrectly on the probe task were also excluded from further analyses because on these trials, participants may not have been attending in the correct location at the beginning of the trial. In addition, in the lowload and high-load conditions, trials in which participants responded incorrectly on the arithmetic task were excluded because the load manipulation may not have been successful. Each participant's mean performance was then calculated for each condition. The percentage of total excluded trials (i.e., combined RT and WM task accuracy exclusions) were $2.7 \%$, $16.5 \%$, and $21.6 \%$ for the no-load, low-load, and high-load conditions, respectively.

Next, the two bias indices were calculated to establish an attentional engagement bias index and an attentional disengagement bias index for each participant in the no-load, lowload, and high-load conditions. These bias scores were subjected to a 2 (trial type: engagement or disengagement bias) $\times$ 3 (load: no, low, and high) ANCOVA, with the continuous predictor variable of social anxiety. Any violations in sphericity were corrected with the Greenhouse-Geisser estimate. This analysis revealed a significant effect for trial type, $F(1$, $89)=14.58, p<.001, \eta_{\mathrm{p}}{ }^{2}=.141$, as the mean engagement bias index was lower $(M=-23.9 \mathrm{~ms})$ than the disengagement bias index $(M=19.1 \mathrm{~ms})$. In addition, a significant effect was found for load, $F(1.77,157.60)=5.54, p=.007, \eta_{\mathrm{p}}{ }^{2}=.059$. The mean bias scores in the no-load, low-load, and high-load conditions were $3.4,8.5$, and -19.2 , respectively. Follow-up analyses, ANCOVAs that compared the mean bias score (averaged over engagement and disengagement) in two levels of load at a time with social anxiety as a covariate, revealed that the bias scores in no-load and low-load conditions did not significantly differ from one another $(F<1)$. However, the no-load bias, $F(1,89)=5.56, p=.021, \eta_{\mathrm{p}}{ }^{2}=.059$, and lowload bias, $F(1,89)=8.22, p=.005, \eta_{\mathrm{p}}{ }^{2}=.085$, were significantly higher than the high-load bias scores.

Finally, and most importantly, a significant interaction between type of trial, load condition, and social anxiety was revealed, $F(1.70,151.64)=5.18, p=.010, \eta_{\mathrm{p}}{ }^{2}=.055$. To further explore this interaction, separate analyses were conducted for the engagement bias and disengagement bias results.

\section{Engagement bias}

An ANCOVA with 3 levels of load (no-load, low-load, and high-load) and a continuous predictor variable of social anxiety was conducted on the engagement bias. This analysis revealed a main effect for load, $F(1.69,150.49)=3.94, p=$ $.028, \eta_{\mathrm{p}}{ }^{2}=.042$. The engagement biases in the no-load, lowload, and high-load conditions were $-17.9,-9.0$, and -44.8 , respectively. Subsequent ANCOVAs comparing engagement biases and two load levels at a time with the social anxiety covariate included revealed that the engagement biases did not significantly differ in the no-load and low-load conditions ( $F$ $<1)$. However, the difference between the engagement biases in the no-load and high-load conditions were trending toward significance, $F(1,89)=3.74, p=.056, \eta_{\mathrm{p}}{ }^{2}=.040$, and the difference between the engagement biases in the low-load and high-load conditions was significant, $F(1,89)=5.53, p=$ $.021, \eta_{\mathrm{p}}{ }^{2}=.059$. This indicates that under high load, the engagement bias was reduced.

In addition, a significant interaction effect was found between load and social anxiety, $F(1.69,150.49)=4.46, p=$ $.018, \eta_{\mathrm{p}}{ }^{2}=.048$. The relationship between social anxiety and the engagement bias was then analyzed separately for the three load conditions using Pearson correlational analyses. For the no-load condition, a significant positive correlation was found between the engagement bias and social anxiety $(r=.26, p=.012)$. This relationship is illustrated in Fig. 2 .

For the low-load condition, the correlation between the engagement bias and social anxiety was not significant $(r=$ $-.05, p=.651)$. This relationship is demonstrated in Fig. 3.

For the high-load condition, the correlation between the engagement bias and social anxiety was not significant $(r=$ $-.18, p=.090)$. This relationship is demonstrated in Fig. 4.

\section{Disengagement bias}

To assess the relationship between social anxiety and the attentional disengagement effect, a three-way (load: no-load, low-load, and high-load) ANCOVA, with the continuous predictor variable of social anxiety, was conducted on the disengagement bias. Neither the main effect of load, $F(1.74$, $154.80)=1.31 p=.270, \eta_{\mathrm{p}}{ }^{2}=.015$, nor the interaction between load and social anxiety, $F(1.74,154.80)=1.74, p=$ $.183, \eta_{\mathrm{p}}^{2}=.019$, were significant.

\section{Gender effect}

Past research has found that face recognition may be affected by the gender of the face and the gender of the observer (e.g., Wells, Gillespie, \& Rotshtein, 2016). For instance, a happy face advantage has been found for female faces (Becker, Kenrick, Neuberg, Blackwell, \& Smith, 2007; Hess, Blairy, \& Kleck, 1997; Tucker \& Riggio, 1988), whereas anger has been found to be more readily recognized for male faces (Becker et al., 2007). Therefore, to test the effect of face and participant gender on bias scores, the bias scores were subjected to a 2 (face gender: male or female) $\times 2$ (participant gender: male or female) $\times 2$ (trial type: engagement or disengagement bias) $\times 3$ (load: no, low, and high) ANCOVA, with the continuous predictor variable of social anxiety. No significant main or interactive effects were observed for face gender ( $p$ s $\geq .318$ ) or participant gender ( $p s \geq .164)$. 


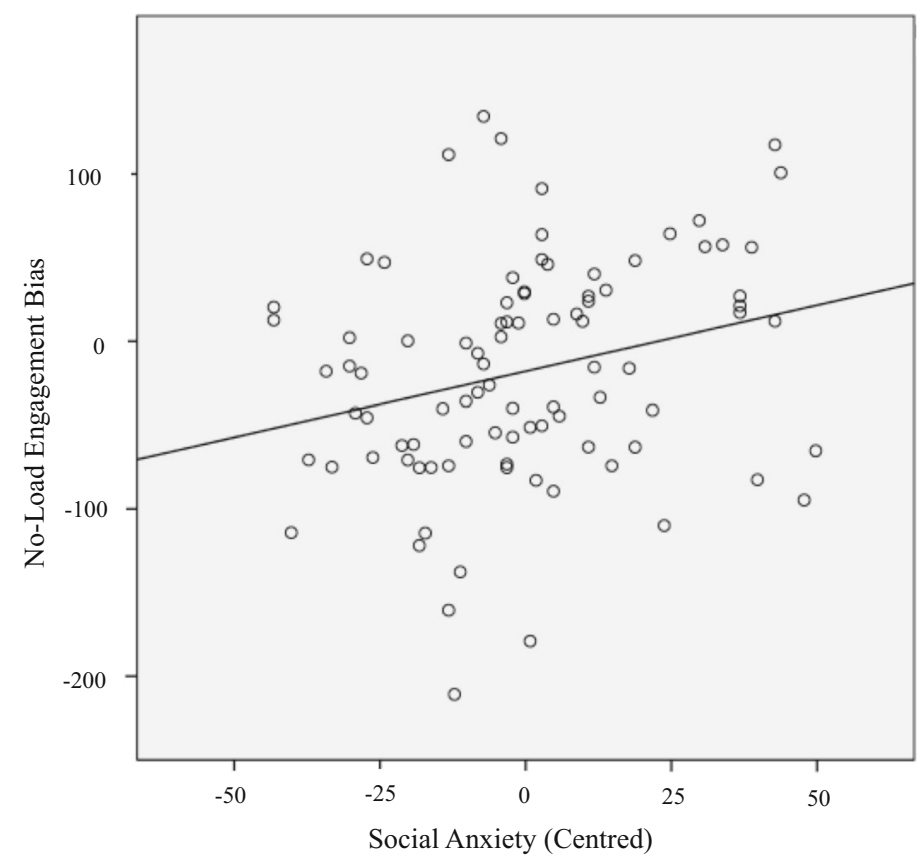

$\mathrm{R}^{2}$ Linear $=0.068$

Fig. 2 Graphical representation of the relationship between social anxiety ( $x$-axis) and the engagement bias to threat ( $y$-axis) under no additional working memory load

\section{Discussion}

The present study sought to further understand the attentional mechanisms underlying social anxiety by testing the role of top-down attention in engaging and disengaging attention from threatening facial expressions. This was achieved by testing engagement and disengagement effects under three WM-load conditions: no-load, low-load (simple arithmetic question), and high-load (difficult arithmetic question) conditions. Social anxiety was not associated with delayed

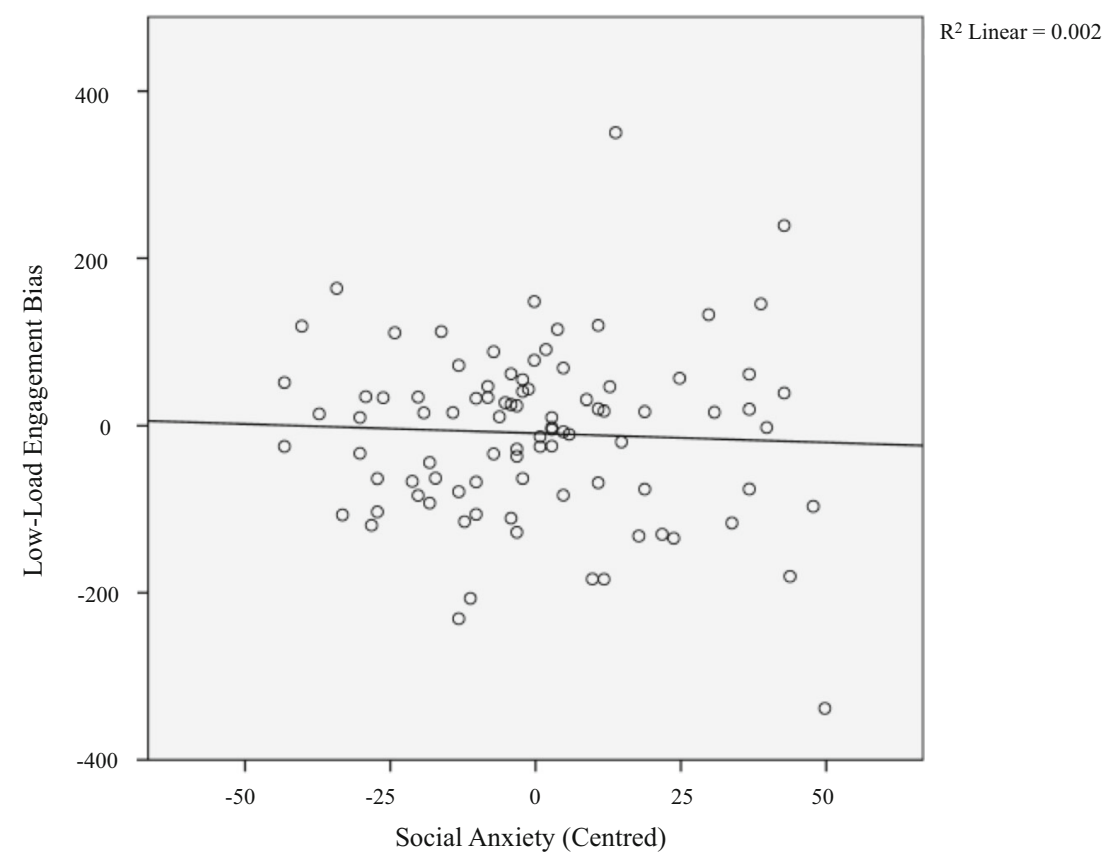

Fig. 3 Graphical representation of the relationship between social anxiety ( $x$-axis) and the engagement bias to threat (y-axis) under low working memory load 


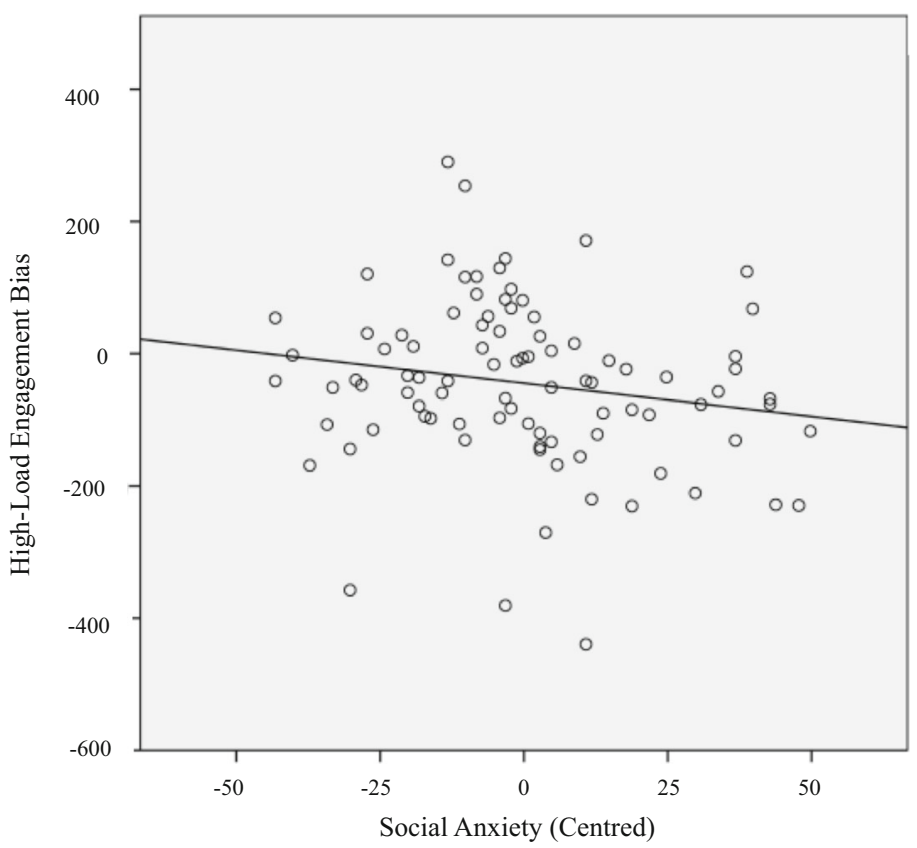

$\mathrm{R}^{2}$ Linear $=0.032$

Fig. 4 Graphical representation of the relationship between social anxiety ( $x$-axis) and the engagement bias to threat (y-axis) under high working memory load.

disengagement from threat. However, higher levels of social anxiety were associated with enhanced engagement with threat. In addition, load moderated the engagement bias, thus implicating the role of top-down attention in this bias.

\section{Social anxiety and an engagement bias toward threat}

In the current study, the results of the no-load condition were a partial replication of Grafton and Macleod's (2016) findings. Grafton and Macleod found that high social anxiety was associated with an engagement bias toward negative facial expressions. The current study found a similar effect, with higher levels of social anxiety associated with an increased engagement bias. A slight difference between the two studies, though, was that the overall means in the current study were negative, not positive. That is, Grafton and Macleod reported a mean engagement bias of $46.67 \mathrm{~ms}$ for participants with high social anxiety, and $12.66 \mathrm{~ms}$ for participants with low social anxiety. When a median split is applied to the current data, participants with high social anxiety have a mean engagement bias of -2.31 , and participants with low social anxiety have a mean engagement bias of $-33.12 \mathrm{~ms}$. On initial consideration, it does not appear that we observed an engagement bias toward threat, as, on average, participants did not respond faster to the probe following the threatening face compared with the neutral face. However, we contend that the current findings reflect the early stages of shifting attention toward the threatening face. That is, participants were generally faster following the neutral face because their attention had been secured by the previously presented cue in that location. Then, as seen in Fig. 2, there is a linear relationship between increased engagement bias scores and social anxiety. In fact, the participants $(n=30)$ scoring above the cutoff for "probable" social anxiety on the LSAS-SR (score of 60) had a mean engagement bias of 3.20. In addition, participants ( $n=$ 10) scoring above the cutoff for "highly probable" social anxiety (score of 90) had a mean engagement bias of 10.89. This indicates that participants with high social anxiety were shifting their attention away from the cued location toward the threatening expression.

Furthermore, although the engagement biases in the current study differ from Grafton and MacLeod's (2016) study, the difference in engagement bias between the low and high social anxiety groups were almost identical. Grafton and Macleod reported engagement bias means of $46.67 \mathrm{~ms}$ for participants with high social anxiety and $12.66 \mathrm{~ms}$ for participants with low social anxiety, which corresponds to a mean difference between groups of $34.01 \mathrm{~ms}$. The mean difference between participants with low and high social anxiety in the current study was $30.81 \mathrm{~ms}$. This indicates that the same pattern of results between participants with low and high social anxiety were found.

The major difference in methodology between the current study and Grafton and MacLeod's study is the presentation times of the faces. Grafton and Macleod (2016) presented the faces for 500-ms and 1,000-ms durations, and found that social anxiety was associated with an engagement bias for the 500 -ms, but not the 1,000-ms duration. The current study used a 200-ms duration. It is, therefore, likely that at this duration participants had only just started to shift their attention from 
the cued neutral face to the threatening face. That is, the bias likely falls within a temporal window; at 200-ms duration, participants with high social anxiety may start shifting their attention away from the neutral face toward the threatening face, then at 500-ms duration, they may have fully engaged attention at the threatening face, and then this bias may no longer be present after $1,000 \mathrm{~ms}$ of duration.

The current study also extended on Grafton and Macleod's (2016) study by testing whether the engagement bias is driven by top-down or bottom-up attention. This was achieved with the addition of a WM load. This had previously been tested by Boal et al. (2018), who did not replicate Grafton and MacLeod's finding of enhanced engagement under the no-load condition. Boal et al. also found no effect of additional WM load on engagement biases. However, there were several limitations undermining Boal et al.' (2018) design, which have been improved in the current study (as discussed in the introduction). Both the current study and Grafton and MacLeod's study found evidence that social anxiety is associated with an engagement bias to threat. One possibility, therefore, is that Boal and colleagues' finding that social anxiety was associated with a reduced bias to threat may be because participants with higher levels of social anxiety had greater attention to the inverted threatening faces. Furthermore, contrary to Boal and colleagues' study, the current study found that the engagement bias to threat for socially anxious participants was eliminated by load. We posit that this discrepancy in results may be because the load task in Boal et al.'s study (digit span) may not have been sufficiently complex to exhaust the participants' WM resources, unlike the arithmetic task in the current study.

Because enhanced attention to threat was eliminated with the addition of load, the results indicate that shifting attention to an angry face for socially anxious individuals is dependent on the availability of WM resources. Thus, the engagement bias toward threat is not automatic, but is instead reliant on top-down attention. Indeed, this bias was eliminated even under low-load. This indicates that the threat bias is only found when individuals have few requirements on WM and they can, thus, allocate those spare resources to attending to threat. This finding challenges the evolutionary perspective that anxiety results in enhanced bottom-up threat detection. LoBue (2014) found that individuals have a bias toward curvilinear lines (representative of snakes) and that anxiety increases this bias. However, LoBue did not employ an additional WM load to test if this bias is bottom up or if it requires WM resources. This raises the possibility that either all engagement threat biases are driven by top-down attention, or although bottomup detection occurs with curvilinear lines, it does not occur with more complex categories such as faces. Further research is needed to test these possibilities.
Social anxiety and a disengagement bias from threat

The disengagement results were consistent with Grafton and MacLeod's (2016) findings, whereby social anxiety was not associated with delayed disengagement effects under no-load conditions. Furthermore, the disengagement bias was not significantly affected by the addition of load. These findings help elucidate the discrepancy found in the attentional literature. That is, past research has found that social anxiety is associated with preferential attention to threat (Mogg \& Bradley, 2002; Mogg, et al., 2004; Pishyar et al., 2004), avoidance of threat (Chen, Ehlers, Clark, \& Mansell, 2002; Mansell, Clark, Ehlers, \& Chen, 1999), or no biases at all (Gotlib et al., 2004; Ononaiye, Turpin, \& Reidy, 2007; Pineles \& Mineka, 2005). As seen from the findings of the current study, this discrepancy in the literature may be better understood if further research separately measures engagement and disengagement biases and considers the impact of WM task demands on attentional biases.

\section{Limitations and further directions}

One potential limitation of this study is that, due to separating the trials into distinct blocks, the load tasks could have induced differences in mood. For example, participants may have felt more anxious in the high-load block than in the noload and low-load blocks. In order to test this explicitly, future research could consider intermixing the trials within the same block. Secondly, the study only included angry-neutral face trials. To ensure that these results specifically reflect a threat bias, it would be necessary to test that the bias is not found for other expressions, such as happy and sad faces.

Thirdly, the present design did not measure the potential effect of inhibition of return from the cued location. It is possible that the engagement bias may be influenced both by participants shifting attention toward the threatening face, as well as inhibition of return to the initially cued location. One method of testing this would be to include neutral-neutral trials. If similar engagement bias scores are found for neutral-neutral trials as for angry-neutral trials, this would indicate that inhibition of return is driving the effect. However, if socially anxious participants have a bias to threatening faces only, this would support the conclusion that social anxiety is associated with an engagement bias to threat.

Note that here we have interpreted the bias scores as reflecting an attentional bias associated with (i.e., enhanced engagement with and delayed disengagement from) the angry faces, with the neutral faces simply serving as the control comparison stimulus. There are very strong theoretical reasons to favour this interpretation, since bias toward threat is at the heart of key models of social anxiety (Rapee \& Heimberg, 1997; Wong \& Rapee, 2016) and there is also empirical support for this notion (Grafton \& MacLeod, 
2016; Mogg et al., 2004; Pishyar et al., 2004). It is therefore legitimate to interpret the biases in the way that we have. However, from an alternative perspective, the pattern of results observed could instead reflect attentional processes driven by the neutral face that was paired with the angry face. Again, there are clear reasons not to favour such an interpretation. However, even if one were to run with this interpretation, our overall conclusion - that the observed bias is modulated by working-memory load — still stands. Since automatic processes are thought to be impervious to concurrent cognitive load, this has clear, novel, and important theoretical implications about the nature of the bias.

In summary, the current study suggests that attentional engagement with threat for individuals with social anxiety is driven by top-down attentional mechanisms. These findings can now be used to inform interventions targeted at reducing social anxiety. Attentional training has been used to attempt to alter cognitive biases to threat. Typically, a dot-probe paradigm is employed with the probe almost always replacing the neutral stimuli, thus training the individual to attend to neutral stimuli rather than threatening stimuli. A recent meta-analysis (Liu et al., 2017) found that attentional training for individuals with social anxiety is associated with a small to medium reduction in the threat bias and a small reduction in symptoms of social anxiety. Based on the results of the current study, it is likely that these changes in attentional biases and symptoms rely on changes in top-down attention, such as cognitive control. Further research could measure if cognitive control moderates the benefits received from attentional training. In addition, individuals with poorer cognitive control could receive training to improve their top-down attention to help them reduce their engagement bias with threat. The results of the current study indicate that further research should focus on targeting the engagement bias toward threat and not the disengagement bias, which does not appear to play a large role for individuals with higher levels of social anxiety.

Acknowledgements This research was supported by an Australian Research Council (ARC) Future Fellowship (FT170100021) awarded to S.C.G.

Open practices statement None of the experiments was preregistered. Raw data are publicly available (see https://osf.io/4yks8/).

\section{References}

Alon, Y., Arad, G., Pine, D. S., \& Bar-Haim, Y. (2019). Statistical learning as a predictor of attention bias modification outcome: A preliminary study among socially anxious patients. Behaviour Research and Therapy, 112, 36-41. doi:https://doi.org/10.1016/j.brat.2018. 11.013

Anderson, S. F., Kelley, K., \& Maxwell, S. E. (2017). Sample-size planning for more accurate statistical power: A method adjusting sample effect sizes for publication bias and uncertainty. Psychological
Science, 28(11), 1547-1562. doi:https://doi.org/10.1177/ 0956797617723724

Antony, M. M., Bieling, P. J., Cox, B. J., Enns, M. W., \& Swinson, R. P. (1998). Psychometric properties of the 42 -item and 21-item versions of the Depression Anxiety Stress Scales in clinical groups and a community sample. Psychological Assessment, 10(2), 176-181. doi:https://doi.org/10.1037/1040-3590.10.2.176

Arnold, D. H., \& Lipp, O. V. (2011). Discrepant integration times for upright and inverted faces. Perception, 40(8), 989-999. doi:https:// doi.org/10.1068/p6955

Baker, S. L., Heinrichs, N., Kim, H. J., \& Hofmann, S. G. (2002). The Liebowitz social anxiety scale as a self-report instrument: A preliminary psychometric analysis. Behaviour Research and Therapy, 40(6), 701-715. doi:https://doi.org/10.1016/S0005-7967(01) 00060-2

Becker, D. V., Kenrick, D. T., Neuberg, S. L., Blackwell, K. C., \& Smith, D. M. (2007). The confounded nature of angry men and happy women. Journal of Personality and Social Psychology, 92(2), 179-190. doi:https://doi.org/10.1037/0022-3514.92.2.179

Boal, H. L., Christensen, B. K., \& Goodhew, S. C. (2018). Social anxiety and attentional biases: A top-down contribution? Attention, Perception, \& Psychophysics, 80(1), 42-53. doi:https://doi.org/10. 3758/s13414-017-1415-5

Brainard, D. H. (1997). The psychophysics toolbox. Spatial Vision, 10(4), 433-436. doi:https://doi.org/10.1163/156856897X00357

Brown, T. A., Chorpita, B. F., Korotitsch, W., \& Barlow, D. H. (1997). Psychometric properties of the Depression Anxiety Stress Scales (DASS) in clinical samples. Behaviour Research and Therapy, 35(1), 79-89. doi:https://doi.org/10.1016/S0005-7967(96)00068-X

Bunge, S. A., Ochsner, K. N., Desmond, J. E., Glover, G. H., \& Gabrieli, J. D. (2001). Prefrontal regions involved in keeping information in and out of mind. Brain, 124(Pt. 10), 2074-2086. doi:https://doi.org/ 10.1093/brain/124.10.2074

Busse, L., Katzner, S., \& Treue, S. (2008). Temporal dynamics of neuronal modulation during exogenous and endogenous shifts of visual attention in macaque area MT. Proceedings of the National Academy of Sciences of the United States of America, 105(42), 16380-16385. doi:https://doi.org/10.1073/pnas.0707369105

Caballo, V. E., Salazar, I. C., Irurtia, M. J., Arias, B., \& Nobre, L. (2013). The assessment of social anxiety through five self-report measures, LSAS-SR, SPAI, SPIN, SPS, AND SIAS: A critical analysis of their factor structure. Behavioral Psychology, 21(3), 423-448.

Chen, Y. P., Ehlers, A., Clark, D. M., \& Mansell, W. (2002). Patients with generalized social phobia direct their attention away from faces. Behaviour Research and Therapy, 40(6), 677-687. doi:https://doi. org/10.1016/S0005-7967(01)00086-9

Cisler, J. M., \& Koster, E. H. (2010). Mechanisms of attentional biases towards threat in anxiety disorders: An integrative review. Clinical Psychology Review, 30(2), 203-216. doi:https://doi.org/10.1016/j. cpr.2009.11.003

Conway, A. R., Kane, M. J., Bunting, M. F., Hambrick, D. Z., Wilhelm, O., \& Engle, R. W. (2005). Working memory span tasks: A methodological review and user's guide. Psychonomic Bulletin \& Review, 12(5), 769-786. doi:https://doi.org/10.3758/BF03196772

Crawford, J. R., \& Henry, J. D. (2003). The Depression Anxiety Stress Scales (DASS): Normative data and latent structure in a large nonclinical sample. British Journal of Clinical Psychology, 42(Pt. 2), 111-131. doi:https://doi.org/10.1348/014466503321903544

de Fockert, J. W., Rees, G., Frith, C. D., \& Lavie, N. (2001). The role of working memory in visual selective attention. Science, 291(5509), 1803-1806. doi:https://doi.org/10.1126/science.1056496

de Gelder, B., Teunisse, J.-P., \& Benson, P. J. (1997). Categorical perception of facial expressions: Categories and their internal structure. Cognition and Emotion, 11(1), 1-23. doi:https://doi.org/10.1080/ 026999397380005 
DeStefano, D., \& LeFevre, J. A. (2004). The role of working memory in mental arithmetic. European Journal of Cognitive Psychology, 16(3), 353-386. doi:https://doi.org/10.1080/09541440244000328

Desimone, R., \& Duncan, J. (1995). Neural mechanisms of selective visual attention. Annual Review of Neuroscience, 18, 193-222. doi:https://doi.org/10.1146/annurev.ne.18.030195.001205

Dima, D. C., Perry, G., Messaritaki, E., Zhang, J., \& Singh, K. D. (2018). Spatiotemporal dynamics in human visual cortex rapidly encode the emotional content of faces. Human Brain Mapping, 39(10), 3993 4006. doi:https://doi.org/10.1002/hbm.24226

Downing, P. E. (2000). Interactions between visual working memory and selective attention. Psychological Science, 11(6), 467-473. doi: https://doi.org/10.1111/1467-9280.00290

Fresco, D. M., Coles, M. E., Heimberg, R. G., Liebowitz, M. R., Hami, S., Stein, M. B., \& Goetz, D. (2001). The Liebowitz Social Anxiety Scale: A comparison of the psychometric properties of self-report and clinician-administered formats. Psychological Medicine, 31(6), 1025-1035. doi:https://doi.org/10.1017/S0033291701004056

Goodhew, S. C., Kendall, W., Ferber, S., \& Pratt, J. (2014). Setting semantics: Conceptual set can determine the physical properties that capture attention. Attention, Perception, \& Psychophysics, 76(6), 1577-1589. doi:https://doi.org/10.3758/s13414-014-0686-3

Gotlib, I. H., Kasch, K. L., Traill, S., Joormann, J., Arnow, B. A., \& Johnson, S. L. (2004). Coherence and specificity of informationprocessing biases in depression and social phobia. Journal of Abnormal Psychology, 113(3), 386-398. doi:https://doi.org/10. 1037/0021-843X.113.3.386

Grafton, B., \& MacLeod, C. (2014). Enhanced probing of attentional bias: The independence of anxiety-linked selectivity in attentional engagement with and disengagement from negative information. Cognition and Emotion, 28(7), 1287-1302. doi:https://doi.org/10. 1080/02699931.2014.881326

Grafton, B., \& MacLeod, C. (2016). Engaging with the wrong people: The basis of selective attention to negative faces in social anxiety. Clinical Psychological Science. doi:https://doi.org/10.1177/ 2167702615616344

Henry, J. D., \& Crawford, J. R. (2005). The short-form version of the Depression Anxiety Stress Scales (DASS-21): Construct validity and normative data in a large non-clinical sample. British Journal of Clinical Psychology, 44(Pt. 2), 227-239. doi:https://doi.org/10. 1348/014466505X29657

Hess, U., Blairy, S., \& Kleck, R. E. (1997). The intensity of emotional facial expressions and decoding accuracy. Journal of Nonverbal Behavior, 21(4), 241-257. doi:https://doi.org/10.1023/A: 1024952730333

Horstmann, G., Lipp, O. V., \& Becker, S. I. (2012). Of toothy grins and angry snarls - Open mouth displays contribute to efficiency gains in search for emotional faces. Journal of Vision, 12(5), 7, 1-15. doi: https://doi.org/10.1167/12.5.7

Jonides, J. (1981). Voluntary versus automatic control over the mind's eye's movement. In J. B. Long \& A. D. Baddeley (Eds.), Attention and performance (Vol. 9, pp. 187-203). Hillsdale, NJ: Erlbaum.

Judah, M. R., Grant, D. M., Lechner, W. V., \& Mills, A. C. (2013). Working memory load moderates late attentional bias in social anxiety. Cognition and Emotion, 27(3), 502-511. doi:https://doi.org/10. 1080/02699931.2012.719490

Kastner, S., \& Pinsk, M. A. (2004). Visual attention as a multilevel selection process. Cognitive, Affective, and Behavioral Neuroscience, 4(4), 483-500. doi:https://doi.org/10.3758/CABN.4.4.483

Kenrick, D. T., Neuberg, S. L., Griskevicius, V., Becker, D. V., \& Schaller, M. (2010). Goal-driven cognition and functional behavior: The fundamental-motives framework. Current Directions in Psychological Science, 19(1), 63-67. doi:https://doi.org/10.1177/ 0963721409359281
Kerr, B. (1973). Processing demands during mental operations. Memory \& Cognition, 1(4), 401-412. doi:https://doi.org/10.3758/ BF03208899

Kondo, H., Osaka, N., \& Osaka, M. (2004). Cooperation of the anterior cingulate cortex and dorsolateral prefrontal cortex for attention shifting. NeuroImage, 23(2), 670-679. doi:https://doi.org/10.1016/ j.neuroimage.2004.06.014

Lang, P. J., Bradley, M. M., \& Cuthbert, B. N. (1997). Motivated attention: Affect, activation and action. In P. J. Lang, R. F. Simons, \& M. T. Balaban (Eds.), Attention and orienting: Sensory and motivational processes (pp. 97-135). Hillsdale, NJ: Erlbaum.

Lavie, N. (2005). Distracted and confused?: Selective attention under load. Trends in Cognitive Sciences, 9(2), 75-82. doi:https://doi. org/10.1016/j.tics.2004.12.004

LeDoux, J. E. (1996). The emotional brain: The mysterious underpinnings of emotional life. New York, NY: Simon \& Schuster.

Levin, J. B., Marom, S., Gur, S., Wechter, D., \& Hermesh, H. (2002). Psychometric properties and three proposed subscales of a selfreport version of the Liebowitz Social Anxiety Scale translated into Hebrew. Depression and Anxiety, 16(4), 143-151. doi:https://doi. org/10.1002/da.10064

Liebowitz, M. R. (1987). Social phobia. Modern Problems of Pharmacopsychiatry, 22, 141-173. doi:https://doi.org/10.1159/ 000414022

Liu, H., Li, X., Han, B., \& Liu, X. (2017). Effects of cognitive bias modification on social anxiety: A meta-analysis. PLOS ONE, 12(4), e0175107. doi:https://doi.org/10.1371/journal.pone.0175107

LoBue, V. (2014). Deconstructing the snake: The relative roles of perception, cognition, and emotion on threat detection. Emotion, 14(4), 701-711. doi:https://doi.org/10.1037/a0035898

LoBue, V., \& DeLoache, J. S. (2011). What's so special about slithering serpents? Children and adults rapidly detect snakes based on their simple features. Visual Cognition, 19(1), 129-143. doi:https://doi. org/10.1080/13506285.2010.522216

LoBue, V., \& Larson, C. L. (2010). What makes an angry face look so ... angry? Examining visual attention to the shape of threat in children and adults. Visual Cognition, 18(8), 1165-1178. doi:https://oi.org/ 10.1080/13506281003783675

LoBue, V., Rakison, D. H., \& DeLoache, J. S. (2010). Threat perception across the life span: Evidence for multiple converging pathways. Current Directions in Psychological Science, 19(6), 375-379. doi: https://doi.org/10.1177/0963721410388801

Lovibond, S. H., \& Lovibond, P. F. (1995). Manual for the depression anxiety stress scales. Sydney, Australia: Psychology Foundation.

Lovibond, S. H., \& Lovibond, P. F. (2005). Manual for the depression anxiety stress scales. Sydney, Australia: Psychology Foundations.

Mansell, W., Clark, D. M., Ehlers, A., \& Chen, Y. P. (1999). Social anxiety and attention away from emotional faces. Cognition and Emotion, 13(6), 673-690. doi:https://doi.org/10.1080/ 026999399379032

Mogg, K., \& Bradley, B. P. (1998). A cognitive-motivational analysis of anxiety. Behaviour Research and Therapy, 36(9), 809-848. doi: https://doi.org/10.1016/S0005-7967(98)00063-1

Mogg, K., \& Bradley, B. P. (2002). Selective orienting of attention to masked threat faces in social anxiety. Behaviour Research and Therapy, 40(12), 1403-1414. doi:https://doi.org/10.1016/S00057967(02)00017-7

Mogg, K., Philippot, P., \& Bradley, B. P. (2004). Selective attention to angry faces in clinical social phobia. Journal of Abnormal Psychology, 113(1), 160-165. doi:https://doi.org/10.1037/0021843X.113.1.160

Most, S. B. (2013). Setting sights higher: Category-level attentional set modulates sustained inattentional blindness. Psychological Research, 77(2), 139-146. doi:https://doi.org/10.1007/s00426-0110379-7 
Oakman, J., Van Ameringen, M., Mancini, C., \& Farvolden, P. (2003). A confirmatory factor analysis of a self-report version of the Liebowitz Social Anxiety Scale. Journal of Clinical Psychology, 59(1), 149161. doi:https://doi.org/10.1002/jclp.10124

Öhman, A. (2007). Has evolution primed humans to "beware the beast"? Proceedings of the National Academy of Sciences of the United States of America, 104(42), 16396-16397. doi:https://doi.org/10. 1073/pnas.0707885104

Ononaiye, M. S. P., Turpin, G., \& Reidy, J. G. (2007). Attentional bias in social anxiety: Manipulation of stimulus duration and socialevaluative anxiety. Cognitive Therapy and Research, 31(6), 727740. doi:https://doi.org/10.1007/s10608-006-9096-8

Pineles, S. L., \& Mineka, S. (2005). Attentional biases to internal and external sources of potential threat in social anxiety. Journal of Abnormal Psychology, 114(2), 314-318. doi:https://doi.org/10. 1037/0021-843x.114.2.314

Pishyar, R., Harris, L. M., \& Menzies, R. G. (2004). Attentional bias for words and faces in social anxiety. Anxiety, Stress and Coping, 17(1), 23-36. doi:https://doi.org/10.1080/10615800310001601458

Qi, S., Zeng, Q., Luo, Y., Duan, H., Ding, C., Hu, W., \& Li, H. (2014). Impact of working memory load on cognitive control in trait anxiety: An ERP study. PLOS ONE, 9(11), e111791. doi:https://doi.org/ 10.1371/journal.pone.0111791

Rapee, R. M., \& Heimberg, R. G. (1997). A cognitive-behavioral model of anxiety in social phobia. Behaviour Research and Therapy, 35(8), 741-756.

Rudaizky, D., Basanovic, J., \& MacLeod, C. (2014). Biased attentional engagement with, and disengagement from, negative information: Independent cognitive pathways to anxiety vulnerability? Cognition and Emotion, 28(2), 245-259. doi:https://doi.org/10.1080/ 02699931.2013 .815154

Rytwinski, N. K., Fresco, D. M., Heimberg, R. G., Coles, M. E., Liebowitz, M. R., Cissell, S., . . . Hofmann, S. G. (2009). Screening for social anxiety disorder with the self-report version of the Liebowitz Social Anxiety Scale. Depression and Anxiety, 26(1), 34-38. doi:https://doi.org/10.1002/da.20503

Sarampalis, A., Kalluri, S., Edwards, B., \& Hafter, E. (2009). Objective measures of listening effort: Effects of background noise and noise reduction. Journal of Speech, Language, and Hearing Research, 52(5), 1230-1240. doi:https://doi.org/10.1044/10924388(2009/08-0111)

Searcy, J. H., \& Bartlett, J. C. (1996). Inversion and processing of component and spatial-relational information in faces. Journal of Experimental Psychology: Human Perception and Performance, 22(4), 904-915. doi:https://doi.org/10.1037/0096-1523.22.4.904

Tabachnick, B. G., \& Fidell, L. S. (2013). Using multivariate statistics (6th ed.). Boston, MA: Pearson Education.
Tucker, J. S., \& Riggio, R. E. (1988). The role of social skills in encoding posed and spontaneous facial expressions. Journal of Nonverbal Behavior, 12(2), 87-97. doi:https://doi.org/10.1007/Bf00986927

Tun, P. A., McCoy, S., \& Wingfield, A. (2009). Aging, hearing acuity, and the attentional costs of effortful listening. Psychology and Aging, 24(3), 761-766. doi:https://doi.org/10.1037/a0014802

Van Dillen, L. F., \& Koole, S. L. (2009). How automatic is "automatic vigilance"? The role of working memory in attentional interference of negative information. Cognition and Emotion, 23(6), 1106-1117. doi:https://doi.org/10.1080/02699930802338178

Wechsler, D. (2008). Wechsler Adult Intelligence Scale - Fourth Edition: Technical and interpretative manual. San Antonio, TX: Pearson Assessment.

Wells, L. J., Gillespie, S. M., \& Rotshtein, P. (2016). Identification of emotional facial expressions: Effects of expression, intensity, and sex on eye gaze. PLOS ONE, 11(12), e0168307. doi:https://doi. org/10.1371/journal.pone.0168307

White, S. W., Capriola-Hall, N. N., Wieckowski, A. T., \& Ollendick, T. H. (2019). Change in gaze-based attention bias in adolescents with Social Anxiety Disorder. Cognition and Emotion, 1-9. Advance online publication. doi:https://doi.org/10.1080/02699931.2019. 1598938

Wieckowski, A. T., Capriola-Hall, N. N., Elias, R., Ollendick, T. H., \& White, S. W. (2019). Variability of attention bias in socially anxious adolescents: Differences in fixation duration toward adult and adolescent face stimuli. Cognition and Emotion, 33(4), 825-831. doi: https://doi.org/10.1080/02699931.2018.1476322

Wong, Q. J. J., \& Rapee, R. M. (2016). The aetiology and maintenance of social anxiety disorder: A synthesis of complimentary theoretical models and formulation of a new integrated model. Journal of Affective Disorders, 203, 84-100. doi:https://doi.org/10.1016/j.jad. 2016.05.069

Wyble, B., Folk, C., \& Potter, M. (2013). Contingent attentional capture by conceptually relevant images. Journal of Experimental Psychology: Human Perception and Performance, 39(3), 861871. doi:https://doi.org/10.1037/a0030517

Young, S. G., Hugenberg, K., Bernstein, M. J., \& Sacco, D. F. (2012). Perception and motivation in face recognition: A critical review of theories of the cross-race effect. Personality and Social Psychology Review, 16(2), 116-142. doi:https://doi.org/10.1177/ 1088868311418987

Publisher's note Springer Nature remains neutral with regard to jurisdictional claims in published maps and institutional affiliations. 